\title{
Variation of the breast cancer susceptibility marker, rs4245739, is associated with differential miRNA binding and MDM4 expression
}

\author{
Sumadi Lukman Anwar ${ }^{1,2^{*}}$, Angel Carlos-Roman ${ }^{3}$, Wahyu Wulaningsih ${ }^{2,4,5}$, \\ Johnathan Watkins ${ }^{2,6}$ \\ ${ }^{1}$ Division of Surgical Oncology, Department of Surgery, Faculty of Medicine \\ Universitas Gadjah Mada, Yogyakarta, Indonesia. \\ 2 PILAR Research Network, 20 Station Road, Cambridge, Cambridgeshire, UK. \\ ${ }^{3}$ Champalimaud Neuroscience Programme, Avenida de Brasilia, Lisbon, Portugal. \\ ${ }^{4}$ Division of Hemato-Oncology Department of Internal Medicine, Faculty of Medicine, \\ Universitas Gadjah Mada, Yogyakarta, Indonesia. \\ ${ }^{5}$ MRC Unit for Lifelong Health and Ageing, University College London, London, UK \\ ${ }^{6}$ Institute for Mathematical and Molecular Biomedicine, King's College London, UK.
}

DOI: http://dx.doi.org/10.19106/JMedScieSup004804201621

\section{ABSTRACT}

A polymorphism of rs 4245739 has been associated to susceptibility of several cancers, including ER-negative breast cancer. rs4245739 is located at the $3^{\prime}$ UTR of MDM4 gene, an oncogene that negatively regulates p53. The polymorphism has been associated to the binding changes of miR-191. We studied the influence of SNP rs4245739 to the binding of microRNAs, expression of microRNAs, and MDM4. Using FindTar software, we detected the potential microRNAs affected by SNP-flanking sequence. The RNA sequencing data from ER-negative breast cancer were collected to compare the expression of miR-184, miR-191, miR-193a, miR378 , and MDM4 in different genotypes. Comparison of ER-negative patients with positive or negative expression of miR-191, as well as the profile of microRNAs (miR-184, miR-191, miR-193a and miR-378 altogether) might differentiate the expression of MDM4 various alleles. In addition, the number of lymphatic nodes affected in the individuals was also found to be significantly reduced in the risk group according to the data obtained by the miRNA profiling method. We concluded that our methods, especially the miRNA profiling approach, are useful in obtaining new molecular and clinical features related to the rs4245739 SNP, a variant located in the 3'UTR of MDM4 gene known to appear in different types of cancer.

Keywords: ER negative breast cancer, rs4245739, microRNA, MDM4, p53 